\title{
Spectrums and Uniform Mean Ergodicity of Weighted Composition Operators on Fock Spaces
}

\author{
Werkaferahu Seyoum $^{1} \cdot$ Tesfa Mengestie $^{2}$ (D)
}

Received: 12 March 2021 / Revised: 17 September 2021 / Accepted: 20 September 2021 /

Published online: 7 October 2021

(c) The Author(s) 2021

\begin{abstract}
For holomorphic pairs of symbols $(u, \psi)$, we study various structures of the weighted composition operator $W_{(u, \psi)} f=u \cdot f(\psi)$ defined on the Fock spaces $\mathcal{F}_{p}$. We have identified operators $W_{(u, \psi)}$ that have power-bounded and uniformly mean ergodic properties on the spaces. These properties are described in terms of easy to apply conditions relying on the values $|u(0)|$ and $\left|u\left(\frac{b}{1-a}\right)\right|$, where $a$ and $b$ are coefficients from linear expansion of the symbol $\psi$. The spectrum of the operators is also determined and applied further to prove results about uniform mean ergodicity.
\end{abstract}

Keywords Fock spaces · Power bounded · Mean ergodic · Compact - Composition · Weighted composition operators $\cdot$ Spectrum

Mathematics Subject Classification Primary: 47B32 - 30H20; Secondary: 46E22 . 46E20 - 47B33

\section{Introduction}

We denote by $\mathcal{H}(\mathbb{C})$ the space of analytic functions on the complex plane $\mathbb{C}$. For pairs of functions $(u, \psi)$ in $\mathcal{H}(\mathbb{C})$, the weighted composition operator $W_{(u, \psi)}$ is defined by $W_{(u, \psi)} f=u \cdot f(\psi), f \in \mathcal{H}(\mathbb{C})$. The operator generalizes both the composition $C_{\psi}$

Communicated by Yoshihiro Sawano.

$\bowtie \quad$ Tesfa Mengestie

Tesfa.Mengestie@hvl.no

Werkaferahu Seyoum

Werkaferahus@gmail.com

1 Department of Mathematics, Kotebe Metropolitan University, Addis Ababa, Ethiopia

2 Mathematics Section, Western Norway University of Applied Sciences, Klingenbergvegen 8, 5414 Stord, Norway 
and multiplication $M_{u}$ operators since it can be factored as $W_{(u, \psi)}=M_{u} C_{\psi}$. Weighted composition operators have been a subject of intense studies in the last several years partly because they found applications in the description of isometries on spaces of analytic functions; see the monographs $[10,11]$ for detailed accounts. For studies on the various properties of the operators, for example, on the classical Fock spaces $\mathcal{F}_{p}$, one may consult the works in $[15,24,25,27]$ and the references therein. Recall that $\mathcal{F}_{p}$ are spaces consisting of all entire functions $f$ for which

$$
\|f\|_{p}=\left\{\begin{array}{l}
\left(\frac{p}{2 \pi} \int_{\mathbb{C}}|f(z)|^{p} e^{-\frac{p|z|^{2}}{2}} d A(z)\right)^{\frac{1}{p}}<\infty, \quad 1 \leq p<\infty \\
\sup _{z \in \mathbb{C}}|f(z)| e^{-\frac{|z|^{2}}{2}}<\infty, \quad p=\infty,
\end{array}\right.
$$

where $d A$ is the Lebesgue area measure on $\mathbb{C}$. For each function $f \in \mathcal{H}(\mathbb{C})$, the subharmonicity of $|f|^{p}$ implies that the local point estimate

$$
|f(z)|^{p} e^{-\frac{p|z|^{2}}{2}} \leq \int_{D(z, 1)}|f(w)|^{p} e^{-\frac{p|w|^{2}}{2}} d A(w)
$$

holds where $D(z, 1)$ is a disc of radius 1 and center $z$. This implies further

$$
|f(z)| \leq\left(\frac{2 \pi}{p}\right)^{\frac{1}{p}} e^{\frac{|z|^{2}}{2}}\|f\|_{p} .
$$

By definition of the norm, the estimate in (1.2) is valid for $p=\infty$ as well.

The space $\mathcal{F}_{2}$ is a reproducing kernel Hilbert space with kernel function $K_{w}(z):=e^{\bar{w} z}$ and normalized kernel $k_{w}:=\left\|K_{w}\right\|_{2}^{-1} K_{w}$. A straightforward calculation shows that $k_{w}$ belongs to all the Fock spaces $\mathcal{F}_{p}$ with a unit norm $\left\|k_{w}\right\|_{p}=1$ for all $w \in \mathbb{C}$.

The rest of the manuscript is organized as follows. In Sect. 2, we study the powerbounded property of the operators. As stated in Theorems 2.1 and 2.3, these properties are described in terms of simple to apply conditions which are merely based on the values of the numbers $|u(0)|$ or $\left|u\left(\frac{b}{1-a}\right)\right|$, where the constants $a$ and $b$ are from the linear expansion of the symbol $\psi(z)=a z+b$. The proofs of the results are presented in Sects. 2.1 and 2.2. In Sect. 3, we identify the spectra of the operators on $\mathcal{F}_{p}$ for all $1 \leq p \leq \infty$; see Theorem 3.1 whose proof is given in Sect. 3.1. Section 4 contains several results on the uniform mean ergodic properties of the operators.

We conclude this section with a word on notation. The notion $U(z) \lesssim V(z)$ (or equivalently $V(z) \gtrsim U(z))$ means that there is a constant $C$ such that $U(z) \leq C V(z)$ holds for all $z$ in the set of a question. We write $U(z) \simeq V(z)$ if both $U(z) \lesssim V(z)$ and $V(z) \lesssim U(z)$.

\section{Power-Bounded $W_{(u, \psi)}$}

We start this section by recalling certain definitions related to dynamics of an operator. Let $T$ be a bounded operator on a Banach space $\mathcal{X}$. Then, we set the operator $T^{n}$ as 
the $n$th iterate of $T ; T^{n}=T \circ T \circ \ldots \circ T n$-times and $T^{0}=I$, where $I$ is the identity map on $\mathcal{X}$. The operator $T$ is said to be power bounded on $\mathcal{X}$ if $\sup _{n \in \mathbb{N}}\left\|T^{n}\right\|<\infty$. Obviously, any operator with norm at most 1 is power bounded. The notion of power boundedness or estimating $\left\|T^{n}\right\|$ plays an important role in the study of numerical stability of initial value problems. If

$$
T_{n}:=\frac{1}{n} \sum_{m=1}^{n} T^{m}, \quad n \in \mathbb{N}
$$

denotes its the $n$th Cesàro means, then $T$ is called mean ergodic if there exists a bounded operator $P$ on $\mathcal{X}$ such that for each $f$ in $\mathcal{X}$

$$
\lim _{n \rightarrow \infty}\left\|T_{n} f-P f\right\|=0,
$$

and uniformly mean ergodic if the pointwise convergence above is uniform;

$$
\lim _{n \rightarrow \infty}\left\|T_{n}-P\right\|=0
$$

A straightforward simplification gives that for each $n \in \mathbb{N}$, the relation

$$
\frac{1}{n} T^{n}=T_{n}-\frac{n-1}{n} T_{n-1}
$$

holds where we set $T_{0}=I$ as the identity operator on $\mathcal{X}$. This immediately implies that if $T$ is mean ergodic, then $\frac{1}{n} T^{n} x \rightarrow 0$ as $n \rightarrow \infty$ for all $x \in \mathcal{X}$. Similarly, $\frac{1}{n}\left\|T^{n}\right\| \rightarrow 0$ whenever $T$ is uniformly mean ergodic. A number of authors have studied ergodicity of operators on various functional spaces; see, for example, $[1,3,5,6]$. The monographs $[14,28]$ provide basic information on ergodic theory. Inspired by all these works, the authors and J. Bonet [26] studied the mean ergodicity of composition operators acting on generalized Fock spaces and concluded that all bounded composition operators on Fock spaces $\mathcal{F}_{p}$ are power bounded whenever $1 \leq p \leq \infty$. In this section, we show that this conclusion is no longer true in general for the weighted composition operators $W_{(u, \psi)}$. It is found that $W_{(u, \psi)}$ is power bounded only when the weight function $u$ satisfies an interesting point value condition as precisely stated in the next two main theorems and proposition.

The study of the dynamics of an operator is related to the study of its iterates. For $f \in \mathcal{H}(\mathbb{C})$, a simple argument shows that the image of $f$ under the iterates of $W_{(u, \psi)}$ has the form

$$
W_{(u, \psi)}^{n} f=f\left(\psi^{n}\right) u_{n}, \quad u_{n}(z):=\prod_{j=0}^{n-1} u\left(\psi^{j}(z)\right)
$$

for each $n \in \mathbb{N}$ and $\psi^{0}=I$ the identity map on $\mathbb{C}$. The equations in (2.1) will be repeatedly used in the sequel. 
We now state the main results on power boundedness. Depending on whether $|a|=$ 1 or $|a|<1$, we give two main results in Theorems 2.1 and 2.3.

Theorem 2.1 Let $1 \leq p \leq \infty, u, \psi \in \mathcal{H}(\mathbb{C})$ and $W_{(u, \psi)}$ be bounded on $\mathcal{F}_{p}$, and hence, $\psi(z)=a z+b, \quad|a| \leq 1$. If $|a|=1$, then the following statements are equivalent.

(i) $W_{(u, \psi)}$ is power bounded on $\mathcal{F}_{p}$;

(ii) $\left(\left\|u_{n}\right\|_{p}\right)_{n}$ is a bounded sequence;

(iii) $|u(0)| \leq e^{-\frac{|b|^{2}}{2}}$. In this case for each $n \in \mathbb{N}$

$$
\left\|W_{(u, \psi)}^{n}\right\|=\left(|u(0)| e^{\frac{|b|^{2}}{2}}\right)^{n} .
$$

It is interesting that we have an easy to apply equivalent conditions for the power boundedness of the weighted composition operators. Part (iii) of the condition is also independent of underlying space or the exponents $p$. In addition, by setting $n=1$, the theorem provides a simple expression for the norm of the operators, namely that

$$
\left\|W_{(u, \psi)}\right\|=|u(0)| e^{\frac{|b|^{2}}{2}} .
$$

We recall that a bounded linear operator is a contraction when its norm is bounded by 1. In view of this, we may add one more equivalent condition to the above list in the theorem, namely that $W_{(u, \psi)}$ is power bounded on $\mathcal{F}_{p}$ if and only if it is a contraction. It should be also noted that for the case $a \neq 1$ and $|a|=1$, the above conditions are also equivalent to $\left|u\left(\frac{b}{1-a}\right)\right| \leq 1$ since an application of Lemma 2.4 implies

$$
\begin{aligned}
\left|u\left(\frac{b}{1-a}\right)\right| & =|u(0)|\left|K_{-\bar{a} b}\left(\frac{b}{1-a}\right)\right|=|u(0)|\left|e^{-\frac{a|b|^{2}}{1-a}}\right| \\
& =|u(0)| e^{-|b|^{2} \Re\left(\frac{a}{1-a}\right)}=|u(0)| e^{\frac{|b|^{2}}{2}},
\end{aligned}
$$

where $\Re$ denotes the real part of the given complex number. This inspires us to ask whether a similar condition works for the remaining case, namely that when $|a|<1$. In this case, as will be explained later, the powers of weighted composition operators are again weighted composition operators. This together with the relations in (2.6) and (2.7) ensures that the following necessary and sufficient conditions hold whenever $|a|<1$.

Proposition 2.2 Let $1 \leq p \leq \infty, u, \psi \in \mathcal{H}(\mathbb{C})$ and $W_{(u, \psi)}$ be bounded on $\mathcal{F}_{p}$. Let $\psi(z)=a z+b$ and $|a|<1$.

(i) If $W_{(u, \psi)}$ is power bounded on $\mathcal{F}_{p}$, then

$$
\left|u\left(\frac{b}{1-a}\right)\right| \leq 1 .
$$


(ii) $W_{(u, \psi)}$ is power bounded on $\mathcal{F}_{p}, p<\infty$ if

$$
\left|u\left(\frac{b}{1-a}\right)\right| \leq|a|^{\frac{2}{p}} .
$$

When $p \rightarrow \infty$, the right-hand side in (2.4) tends to 1 . Thus, the condition in (2.3) is both necessary and sufficient for $W_{(u, \psi)}$ to be power bounded on the space $\mathcal{F}_{\infty}$. In particular, when $W_{(u, \psi)}$ is compact, we record our next main result which holds true on all the spaces $\mathcal{F}_{p}$.

Theorem 2.3 Let $1 \leq p \leq \infty, u, \psi \in \mathcal{H}(\mathbb{C})$ and $W_{(u, \psi)}$ be bounded on $\mathcal{F}_{p}$, and $\psi(z)=a z+b$, with $|a|<1$. Let $u$ be non-vanishing and $W_{(u, \psi)}$ be compact. Then, the following statements are equivalent.

(i) $W_{(u, \psi)}$ is power bounded on $\mathcal{F}_{p}$;

(ii) $\left(\left\|u_{n}\right\|_{p}\right)_{n}$ is a bounded sequence;

(iii) $\left|u\left(\frac{b}{1-a}\right)\right| \leq 1$.

As in Theorem 2.1, part (iii) of the condition is simple to apply and independent of the exponents $p$. Observe that from the two theorems above, it is easy to see that a bounded composition operator $C_{\psi}$ is always power bounded, while the multiplication operator $M_{u}$ is not in general; see Corollary 4.7.

To prove the results, we need to make some preparations. The bounded and compact weighted composition operators on Fock spaces were characterized first in terms of Berezin-type integral transforms in [24,25,27]. Later, Le [15] considered the Hilbert space $\mathcal{F}_{2}$ setting and obtained a simpler condition, namely that $W_{(u, \psi)}$ is bounded on $\mathcal{F}_{2}$ if and only if

$$
M(u, \psi):=\sup _{z \in \mathbb{C}}|u(z)| e^{\frac{1}{2}\left(|\psi(z)|^{2}-|z|^{2}\right)}<\infty
$$

He further proved that (2.5) implies $\psi(z)=a z+b$ with $|a| \leq 1$. In [22], T. Mengestie and M. Worku proved that the Berezin-type integral condition used to describe the boundedness of generalized Volterra-type integral operators $V_{(g, \psi)}$ on the Fock spaces $\mathcal{F}_{p}$ is equivalent to a simple condition as in (2.5). Because of the Littlewood-Paleytype description of the Fock spaces, by simply replacing $\left|g^{\prime}(z)\right| /(1+|z|)$ by $|u(z)|$ in the results there, it has been known that (2.5) in fact describes the bounded weighted composition operators on all the spaces $\mathcal{F}_{p}, 1 \leq p<\infty$, with norm bounds

$$
M(u, \psi) \leq\left\|W_{(u, \psi)}\right\| \leq|a|^{-\frac{2}{p}} M(u, \psi)
$$

For $p=\infty$, the corresponding relation holds with equality,

$$
\left\|W_{(u, \psi)}\right\|=M(u, \psi)
$$

As indicated in [15], an interesting consequence of (2.5) is that if $|a|=1$, then a simple argument with Liouville's theorem gives that the weight function $u$ has the 
form $u(z)=u(0) K_{-\bar{a} b}(z)$. This representation of $u$ will play an important role in the rest of the paper. Thus, we may formulate it as a lemma for the purpose of easy further referencing.

Lemma 2.4 Let $1 \leq p \leq \infty, u, \psi \in \mathcal{H}(\mathbb{C})$ and $W_{(u, \psi)}$ be bounded on $\mathcal{F}_{p}$, and hence $\psi(z)=a z+b,|a| \leq 1$. If $|a|=1$, then

$$
u(z)=u(0) K_{-\bar{a} b}(z)
$$

It should be noted that by condition (2.5) it is possible for $W_{(u, \psi)}=M_{u} C_{\psi}$ to be bounded even if both the factors $C_{\psi}$ and $M_{u}$ are unbounded. The functions $u(z)=z$ and $\psi(z)=z+1$ provide such an example.

Similarly, compactness of $W_{(u, \psi)}$ has been described by the fact that $\psi(z)=a z+$ $b,|a| \leq 1$ and $|u(z)| e^{\frac{1}{2}\left(|\psi(z)|^{2}-|z|^{2}\right)} \rightarrow 0$ as $|z| \rightarrow \infty$. The latter condition implies that $|a|<1$ but not conversely. Very recently, Carroll and Gilmore [7] used the idea of order of analytic function and proved the following analogues result.

Lemma 2.5 Let $1 \leq p \leq \infty, u, \psi \in \mathcal{H}(\mathbb{C})$ and $\psi(z)=a z+b,|a|<1$, and assume that $u$ is non-vanishing. Then, $W_{(u, \psi)}$ is compact on $\mathcal{F}_{p}$ if and only if $u$ has the form

$$
u(z)=e^{a_{0}+a_{1} z+a_{2} z^{2}}
$$

for some constants $a_{0}, a_{1}, a_{2}$ such that $\left|a_{2}\right|<\frac{1-|a|^{2}}{2}$.

Next, we consider the following key necessary conditions for power bounded $W_{(u, \psi)}$. The lemma gives a good restriction on the growth of the sequence $\left(\left\|u_{n}\right\|_{p}\right)_{n}$ and the value $\left|u\left(z_{0}\right)\right|$, where $z_{0}$ is a fixed point of $\psi$.

Lemma 2.6 Let $1 \leq p \leq \infty$ and $u, \psi \in \mathcal{H}(\mathbb{C})$. If $W_{(u, \psi)}$ is power bounded on $\mathcal{F}_{p}$, then

(i) $\left|u\left(z_{0}\right)\right| \leq 1$ where $z_{0}$ is a fixed point of $\psi$ :

(ii) $\left(\left\|u_{n}\right\|_{p}\right)_{n}$ is a bounded sequence.

Proof (i). Since the constant function $\mathbf{1}$ belongs to the spaces $\mathcal{F}_{p}$ with $\|\mathbf{1}\|_{p}=1$, using the pointwise estimate in (1.2)

$$
\left\|W_{(u, \psi)}^{n}\right\| \geq\left\|W_{(u, \psi)}^{n} \mathbf{1}\right\|_{p} \gtrsim\left|W_{(u, \psi)}^{n} \mathbf{1}\left(z_{0}\right)\right| e^{-\frac{\left|z_{0}\right|^{2}}{2}}=\left|u_{n}\left(z_{0}\right)\right| e^{-\frac{\left|z_{0}\right|^{2}}{2}}=\left|u\left(z_{0}\right)\right|^{n} e^{-\frac{\left|z_{0}\right|^{2}}{2}}
$$

from which the inequalities

$$
\infty>\sup _{n \in \mathbb{N}}\left\|W_{(u, \psi)}^{n}\right\| \geq e^{-\frac{\left|z_{0}\right|^{2}}{2}} \sup _{n \in \mathbb{N}}\left|u\left(z_{0}\right)\right|^{n}
$$

hold only if $\left|u\left(z_{0}\right)\right| \leq 1$.

To prove (ii), for $p=\infty$ arguing as above we have

$$
\left\|W_{(u, \psi)}^{n}\right\| \geq\left\|W_{(u, \psi)}^{n} \mathbf{1}\right\|_{\infty} \geq\left|W_{(u, \psi)}^{n} \mathbf{1}(z)\right| e^{-\frac{|z|^{2}}{2}}=\left|u_{n}(z)\right| e^{-\frac{|z|^{2}}{2}} .
$$


Taking the supremum with respect to first with $z$ and then with $n$ gives the required assertion. On the other hand, if $p<\infty$, then

$$
\left\|W_{(u, \psi)}^{n}\right\|^{p} \geq\left\|W_{(u, \psi)}^{n} \mathbf{1}\right\|_{p}^{p}=\frac{p}{2 \pi} \int_{\mathbb{C}}\left|u_{n}(z)\right|^{p} e^{-\frac{p|z|^{2}}{2}} d A(z)=\left\|u_{n}\right\|_{p}^{p}
$$

from which the conclusion follows again.

The next simple lemma will be crucial in the proof of Theorem 2.3.

Lemma 2.7 Let $a \in \mathbb{C}$ and $|a|<1$. Then, for all $n \in \mathbb{N}$

$$
\frac{\left|1-a^{2}\right|}{1-|a|^{2}} \geq \frac{\left|1-a^{2 n}\right|}{1-|a|^{2 n}}
$$

Proof Applying triangular inequality,

$$
\begin{aligned}
\frac{\left|1-a^{2 n}\right|}{\left|1-a^{2}\right|} & =\left|1+a^{2}+\left(a^{2}\right)^{2}+\ldots+\left(a^{2}\right)^{n-1}\right| \leq 1+\left|a^{2}\right|+\left|\left(a^{2}\right)^{2}\right|+\ldots+\left|\left(a^{2}\right)^{(n-1)}\right| \\
& =1+|a|^{2}+\left(|a|^{2}\right)^{2}+\ldots+\left(|a|^{2}\right)^{n-1}=\frac{1-|a|^{2 n}}{1-|a|^{2}}
\end{aligned}
$$

from which (2.9) follows.

We are now ready to give the proofs of the previous two main results.

\subsection{Proof of Theorem 2.1}

The statement (i) implies (ii), which is proved in Lemma 2.6. On the other hand, if (ii) holds, then using (1.2)

$$
\infty>\sup _{n \in \mathbb{N}_{0}}\left\|u_{n}\right\|_{p} \gtrsim \sup _{n \in \mathbb{N}_{0}}\left|u_{n}(z)\right| e^{-\frac{|z|^{2}}{2}}
$$

for each $z \in \mathbb{C}$. If $a=1$, then $\psi^{j}(z)=z+j b$ and using Lemma 2.4,

$$
u_{n}(z)=u(0)^{n} \prod_{j=0}^{n-1} K_{-b}(z+j b)=u(0)^{n} e^{l_{n}(z)}
$$

where

$$
l_{n}(z):=-\bar{b} \sum_{j=0}^{n-1}(z+j b)=-\bar{b} n z-\frac{|b|^{2}}{2} n(n-1) .
$$


It follows that

$$
u_{n}(z)=u(0)^{n} e^{-\frac{|b|^{2}}{2} n(n-1)} K_{-n b}(z)
$$

for all $z \in \mathbb{C}$. Considering (2.11) and applying the estimate in (2.10) at $z=-n b$

$$
\begin{aligned}
\sup _{n \in \mathbb{N}_{0}}\left\|u_{n}\right\|_{p} & \gtrsim \sup _{n \in \mathbb{N}_{0}}\left|u_{n}(-n b)\right| e^{-\frac{|n b|^{2}}{2}} \\
& =\sup _{n \in \mathbb{N}_{0}}\left|u(0) e^{\frac{|b|^{2}}{2}}\right|^{n} e^{-|b|^{2} n^{2}} K_{-n b}(-n b)=\sup _{n \in \mathbb{N}_{0}}\left|u(0) e^{\frac{|b|^{2}}{2}}\right|^{n}
\end{aligned}
$$

and hence the statement in (iii) follows. On the other hand, if $a \neq 1$ and $|a|=1$, then set $z_{0}$ be the fixed point of $\psi$ and eventually applying Lemma 2.4

$$
\begin{aligned}
\left|u_{n}\left(z_{0}\right)\right| & =\left|u(0) K_{-\bar{a} b}\left(\frac{b}{1-a}\right)\right|^{n}=\left|u(0) e^{-a \bar{b}\left(\frac{b}{1-a}\right)}\right|^{n} \\
& =|u(0)|^{n} e^{-n \Re\left(\frac{a|b|^{2}}{1-a}\right)}=|u(0)|^{n} e^{\frac{n|b|^{2}}{2}}
\end{aligned}
$$

and the conclusion follows after taking this in (2.10) again.

It remains to prove (iii) implies (i). First, observe that for each $n \in \mathbb{N}$, the operator $W_{(u, \psi)}^{n}$ itself is a weighted composition operator and $W_{(u, \psi)}^{n}=M_{u_{n}} C_{\psi^{n}}=W_{\left(u_{n}, \psi^{n}\right)}$. Then, $W_{(u, \psi)}$ is power bounded if and only if

$$
\sup _{n \in \mathbb{N}} \sup _{z \in \mathbb{C}}\left|u_{n}(z)\right| e^{\frac{1}{2}\left(\left|a^{n} z+\frac{b\left(1-a^{n}\right)}{1-a}\right|^{2}-|z|^{2}\right)}<\infty .
$$

Thus, for $|a|=1$, we apply (2.6) and obtain the norm

$$
\left\|W_{(u, \psi)}^{n}\right\|=\sup _{z \in \mathbb{C}}\left|u_{n}(z)\right| e^{\frac{1}{2}\left(\left|\psi^{n}(z)\right|^{2}-|z|^{2}\right)} .
$$

Our next task is to simplify (2.12). If $a=1$, then the representation in (2.11) implies

$$
\begin{aligned}
\left\|W_{(u, \psi)}^{n}\right\| & =\sup _{z \in \mathbb{C}} e^{\frac{1}{2}\left(|z+n b|^{2}-|z|^{2}\right)}|u(0)|^{n} e^{-\frac{|b|^{2}}{2} n(n-1)}\left|K_{-n b}(z)\right| \\
& =\left(|u(0)| e^{\frac{|b|^{2}}{2}}\right)^{n} \sup _{z \in \mathbb{C}} e^{\Re(n b \bar{z})}\left|K_{-n b}(z)\right|=\left(|u(0)| e^{\frac{|b|^{2}}{2}}\right)^{n}
\end{aligned}
$$

from which the statement follows. 
Next, assume $a \neq 1$ and $|a|=1$. Then, $\psi^{j}(z)=a^{j} z+b \frac{1-a^{j}}{1-a}$. By using Lemma 2.4 again $u_{n}(z)=u(0)^{n} e^{h_{n}(z)}$ where

$$
h_{n}(z):=-a \bar{b} \sum_{j=0}^{n-1}\left(a^{j} z+b \frac{1-a^{j}}{1-a}\right)=-a \bar{b} z \frac{1-a^{n}}{1-a}-\frac{a|b|^{2} n}{1-a}+\frac{a|b|^{2}\left(1-a^{n}\right)}{(1-a)^{2}} .
$$

Thus, we have

$$
u_{n}(z)=u(0)^{n} e^{-\frac{a|b|^{2} n}{1-a}+\frac{a|b|^{2}\left(1-a^{n}\right)}{(1-a)^{2}}} K_{-\bar{a} b \frac{1-\bar{a}^{n}}{1-\bar{a}}}(z)
$$

from which and (2.12)

$$
\left\|W_{(u, \psi)}^{n}\right\|=\sup _{z \in \mathbb{C}} e^{\frac{1}{2}\left|a^{n} z+b \frac{1-a^{n}}{1-a}\right|^{2}-\frac{1}{2}|z|^{2}}|u(0)|^{n}\left|e^{-a \bar{b} z \frac{1-a^{n}}{1-a}-\frac{a|b|^{2} n}{1-a}+\frac{a|b|^{2}\left(1-a^{n}\right)}{(1-a)^{2}}}\right|
$$

where

$$
\left|a^{n} z+b \frac{1-a^{n}}{1-a}\right|^{2}-|z|^{2}=|b|^{2}\left|\frac{1-a^{n}}{1-a}\right|^{2}+2 \Re\left(a^{n} z \bar{b} \frac{1-\bar{a}^{n}}{1-\bar{a}}\right)
$$

and

$$
\left|e^{-a \bar{b} z \frac{1-a^{n}}{1-a}-\frac{a|b|^{2} n}{1-a}+\frac{a|b|^{2}\left(1-a^{n}\right)}{(1-a)^{2}}}\right|=e^{\Re\left(-a \bar{b} z \frac{1-a^{n}}{1-a}-\frac{a|b|^{2} n}{1-a}+\frac{a|b|^{2}\left(1-a^{n}\right)}{(1-a)^{2}}\right)} .
$$

On the other hand,

$$
a^{n} z \bar{b} \frac{1-\bar{a}^{n}}{1-\bar{a}}-a \bar{b} z \frac{1-a^{n}}{1-a}=z \bar{b}\left(a^{n}-1\right)\left(\frac{1}{1-\bar{a}}+\frac{a}{1-a}\right)=0
$$

and combining all the above

$$
\begin{aligned}
\left\|W_{(u, \psi)}^{n}\right\| & =|u(0)|^{n} e^{\frac{|b|^{2}}{2}\left|\frac{1-a^{n}}{1-a}\right|^{2}+\Re\left(\frac{a|b|^{2}(1-a n)}{(1-a)^{2}}-\frac{a|b|^{2} n}{1-a}\right)} \\
& \leq|u(0)|^{n} e^{\frac{|b|^{2}}{2}\left|\frac{2}{1-a}\right|^{2}+\Re\left(\frac{a|b|^{2}\left(1-a^{n}\right)}{(1-a)^{2}}\right)-\Re\left(\frac{a|b|^{2} n}{1-a}\right)} \\
& \leq|u(0)|^{n} e^{\frac{|b|^{2}}{2}\left|\frac{2}{1-a}\right|^{2}+\left|\frac{a|b|^{2}\left(1-a^{n}\right)}{(1-a)^{2}}\right|} e^{-n|b|^{2} \Re\left(\frac{a}{1-a}\right)} \\
& \leq e^{\frac{|b|^{2}}{2}\left|\frac{2}{1-a}\right|^{2}+\frac{2|b|^{2}}{|1-a|^{2}}}\left(|u(0)| e^{-|b|^{2} \Re\left(\frac{a}{1-a}\right)}\right)^{n} \\
& =e^{\frac{|b|^{2}}{2}\left|\frac{2}{1-a}\right|^{2}+\frac{2|b|^{2}}{|1-a|^{2}}}\left(|u(0)| e^{\frac{|b|^{2}}{2}}\right)^{n}
\end{aligned}
$$

Thus, power boundedness follows whenever $|u(0)| \leq e^{-\frac{|b|^{2}}{2}}$. 


\subsection{Proof of Theorem 2.3}

The statement (i) implies (ii), which follows from Lemma 2.6 again. Assuming (ii), we proceed to show that (iii) holds. Using (1.2), we estimate

$$
\infty>\sup _{n \in \mathbb{N}_{0}}\left\|u_{n}\right\|_{p} \gtrsim \sup _{n \in \mathbb{N}_{0}}\left|u_{n}\left(z_{0}\right)\right| e^{-\frac{\left|z_{0}\right|^{2}}{2}},
$$

where $z_{0}=b /(1-a)$ is the fixed point of $\psi$. Moreover, observe that

$$
\left|u_{n}\left(z_{0}\right)\right|=\prod_{j=0}^{n-1} u\left(\psi^{j}\left(z_{0}\right)\right)=\left|u\left(z_{0}\right)\right|^{n}
$$

which together with Lemma 2.4 and (2.15) gives statement (iii).

Next, we prove (iii) implies (i). If $p=\infty$, applying the relation in (2.7) for the weighted composition operator $W_{\left(u_{n}, \psi^{n}\right)}$ we get

$$
\left\|W_{\left(u_{n}, \psi^{n}\right)}\right\|=\left\|W_{(u, \psi)}^{n}\right\|=\sup _{z \in \mathbb{C}}\left|u_{n}(z)\right| e^{\frac{1}{2}\left(\left|a^{n} z+z_{0} b\left(1-a^{n}\right)\right|^{2}-|z|^{2}\right)} .
$$

Thus, $W_{\left(u_{n}, \psi^{n}\right)}$ is power bounded on $\mathcal{F}_{\infty}$ if and only if

$$
\sup _{n \in \mathbb{N}} \sup _{z \in \mathbb{C}}\left|u_{n}(z)\right| e^{\frac{1}{2}\left(\left|a^{n} z+z_{0} b\left(1-a^{n}\right)\right|^{2}-|z|^{2}\right)}<\infty
$$

Therefore, by using the assumption $\left|u\left(\frac{b}{1-a}\right)\right| \leq 1$, we plan to show that (2.16) holds. First, we consider Lemma 2.5 and compute

$$
u_{n}(z)=\prod_{j=0}^{n-1} u\left(\psi^{j}(z)\right)=e^{S_{n}(z)}
$$

where

$$
\begin{aligned}
S_{n}(z)= & \sum_{j=0}^{n-1}\left(a_{0}+a_{1}\left(a^{j} z+\frac{\left(1-a^{j}\right) b}{1-a}\right)+a_{2}\left(a^{j} z+\frac{\left(1-a^{j}\right) b}{1-a}\right)^{2}\right) \\
= & n a_{0}+\frac{a_{1} b n}{1-a}-\frac{a_{1} b\left(1-a^{n}\right)}{(1-a)^{2}}+\frac{a_{1}\left(1-a^{n}\right)}{1-a} z+\frac{a_{2}\left(1-a^{2 n}\right)}{1-a^{2}} z^{2} \\
& +\frac{a_{2} b^{2}}{(1-a)^{2}}\left(n-\frac{2\left(1-a^{n}\right)}{1-a}+\frac{1-a^{2 n}}{1-a^{2}}\right)+\frac{2 a_{2} z b}{1-a}\left(\frac{1-a^{n}}{1-a}-\frac{1-a^{2 n}}{1-a^{2}}\right)
\end{aligned}
$$




$$
\begin{aligned}
= & n a_{0}+\frac{a_{1} b n}{1-a}-\frac{a_{1} b\left(1-a^{n}\right)}{(1-a)^{2}}+\frac{a_{2} b^{2}}{(1-a)^{2}}\left(n-\frac{2\left(1-a^{n}\right)}{1-a}+\frac{1-a^{2 n}}{1-a^{2}}\right) \\
& +\left(\frac{a_{1}\left(1-a^{n}\right)}{1-a}+\frac{2 a_{2} b}{1-a}\left(\frac{1-a^{n}}{1-a}-\frac{1-a^{2 n}}{1-a^{2}}\right)\right) z+\frac{a_{2}\left(1-a^{2 n}\right)}{1-a^{2}} z^{2} .
\end{aligned}
$$

Now taking this into account and the fact that $W_{(u, \psi)}^{n}=W_{\left(u_{n}, \psi^{n}\right)}$, the corresponding notation in (2.5) becomes

$$
\begin{aligned}
M\left(u_{n}, \psi^{n}\right) & =\sup _{z \in \mathbb{C}}\left|u_{n}(z)\right| e^{\frac{1}{2}\left(\left|a^{n} z+\frac{b\left(1-a^{n}\right)}{1-a}\right|^{2}-|z|^{2}\right)} \\
& =e^{c_{n}} \sup _{z \in \mathbb{C}} e^{\Re\left(t_{n} z\right)+\Re\left(p_{n} z^{2}\right)-q_{n}|z|^{2}} \\
& \leq e^{c_{n}} \sup _{z \in \mathbb{C}} e^{\Re\left(t_{n} z\right)+\left(\left|p_{n}\right|-q_{n}\right)|z|^{2}}
\end{aligned}
$$

where $c_{n}$ is the real part of the expression

$$
\begin{aligned}
& n a_{0}+\frac{a_{1} b n}{1-a}-\frac{a_{1} b\left(1-a^{n}\right)^{2}}{1-a}+\frac{a_{2} b^{2}}{(1-a)^{2}}\left(n-\frac{2\left(1-a^{n}\right)}{1-a}+\frac{1-a^{2 n}}{1-a^{2}}\right)+\left|\frac{b\left(1-a^{n}\right)}{1-a}\right|^{2}, \\
& t_{n}=\frac{a_{1}\left(1-a^{n}\right)}{1-a}+\frac{2 a_{2} b}{1-a}\left(\frac{1-a^{n}}{1-a}-\frac{1-a^{2 n}}{1-a^{2}}\right)+\frac{\overline{\left(1-a^{n}\right) b}}{1-\bar{a}} a^{n}, \\
& p_{n}=\frac{a_{2}\left(1-a^{2 n}\right)}{1-a^{2}}, \text { and } q_{n}=\frac{1-|a|^{2 n}}{2} .
\end{aligned}
$$

Now to estimate the supremum in (2.18), we claim that

$$
\left|p_{n}\right|-q_{n}=\left|a_{2}\right|\left|\frac{1-a^{2 n}}{1-a^{2}}\right|-\frac{1-|a|^{2 n}}{2}<0 .
$$

Observe that the inequality holds if and only if

$$
\left|a_{2}\right|<\frac{\left(1-|a|^{2 n}\right)}{\left|1-a^{2 n}\right|} \frac{\left|1-a^{2}\right|}{2} .
$$

This follows immediately from Lemma 2.7 as $\left|a_{2}\right|<\frac{1-|a|^{2}}{2}$.

It follows from this and (2.18) that

$$
M\left(u_{n}, \psi^{n}\right) \lesssim e^{c_{n}} .
$$

On the other hand, since $|a|<1$

$$
c_{n} \leq n \Re\left(a_{0}+\frac{a_{1} b}{1-a}+\frac{a_{2} b^{2}}{(1-a)^{2}}\right)+C
$$


for some positive constant $C$ and hence

$$
e^{c_{n}} \lesssim e^{n \Re\left(a_{0}+\frac{a_{1} b}{1-a}+\frac{a_{2} b^{2}}{(1-a)^{2}}\right)} \simeq\left|u\left(\frac{b}{1-a}\right)\right|^{n}
$$

from which and the assumption that $\left|u\left(\frac{b}{1-a}\right)\right| \leq 1$, the condition in (2.16) follows.

Next, we consider the case when $p<\infty$ and consider first the case $a=0$. Then, $\psi^{n}(z)=b, u_{n}(z)=u(z)(u(b))^{n-1}$ and applying (1.2),

$$
\begin{aligned}
\left\|W_{(u, \psi)}^{n} f\right\|_{p}^{p} & =\frac{p}{2 \pi} \int_{\mathbb{C}}|f(b)|^{p}\left|u_{n}(z)\right|^{p} e^{-\frac{p}{2}|z|^{2}} d A(z) \\
& =\frac{p}{2 \pi} \int_{\mathbb{C}}|f(b)|^{p}|u(z)|^{p}|u(b)|^{(n-1) p} e^{-\frac{p}{2}|z|^{2}} d A(z) \\
& =|f(b)|^{p}|(u(b))|^{(n-1) p}\|u\|_{p}^{p} \leq|(u(b))|^{(n-1) p}\|u\|_{p}^{p} e^{|b|^{2}\|f\|_{p}^{p},}
\end{aligned}
$$

from which we arrive at the claim. Here, note that since $W_{(u, \psi)}$ is bounded, the multiplier $u$ belongs to $\mathcal{F}_{p}$ for all $p$.

If $a \neq 0$, then applying the local point estimate in (1.1),

$$
\begin{aligned}
\left\|W_{(u, \psi)}^{n} f\right\|_{p}^{p}= & \frac{p}{2 \pi} \int_{\mathbb{C}}\left|f\left(a^{n} z+\frac{b\left(1-a^{n}\right)}{1-a}\right)\right|^{p}\left|u_{n}(z)\right|^{p} e^{-\frac{p}{2}|z|^{2}} d A(z) \\
\leq & \frac{p}{2 \pi} \int_{\mathbb{C}} e^{\frac{p}{2}\left(\left|a^{n} z+\frac{b\left(1-a^{n}\right)}{1-a}\right|^{2}-|z|^{2}\right)}\left|u_{n}(z)\right|^{p} \\
& \times \int_{D\left(a^{n} z+\frac{b\left(1-a^{n}\right)}{1-a}, 1\right)}|f(w)|^{p} e^{-\frac{p}{2}|w|^{2}} d A(w) d A(z) \\
= & \frac{p}{2 \pi} \int_{\mathbb{C}} \int_{\mathbb{C}} e^{\frac{p}{2}\left(\left|a^{n} z+\frac{b\left(1-a^{n}\right)}{1-a}\right|^{2}-|z|^{2}\right)}\left|u_{n}(z)\right|^{p} \\
& \chi_{D\left(a^{n} z+\frac{b\left(1-a^{n}\right)}{1-a}, 1\right)}(w)|f(w)|^{p} e^{-\frac{p}{2}|w|^{2}} d A(w) d A(z) .
\end{aligned}
$$

Observe that if $w \in D\left(a^{n} z+\frac{b\left(1-a^{n}\right)}{1-a}, 1\right)$, then

$$
\begin{aligned}
1 & \geq\left|w-a^{n} z-\frac{b\left(1-a^{n}\right)}{1-a}\right|=|a|^{n}\left|\frac{w}{a^{n}}-z-\frac{b\left(1-a^{n}\right)}{a^{n}(1-a)}\right| \\
& =|a|^{n}\left|z-\left(\frac{w}{a^{n}}-\frac{b\left(1-a^{n}\right)}{a^{n}(1-a)}\right)\right|,
\end{aligned}
$$

which holds true if and only if

$$
\frac{1}{|a|^{n}} \geq\left|z-\left(\frac{w}{a^{n}}-\frac{b\left(1-a^{n}\right)}{a^{n}(1-a)}\right)\right| .
$$


Thus, $w$ belongs to the disc

$$
D\left(a^{n} z+\frac{b\left(1-a^{n}\right)}{1-a}, 1\right)
$$

if and only if $z$ belongs to

$$
D\left(\frac{w}{a^{n}}-\frac{b\left(1-a^{n}\right)}{1-a}, \frac{1}{|a|^{n}}\right) .
$$

Making use of this and Fubini's theorem in (2.20)

$$
\begin{aligned}
\left\|W_{(u, \psi)}^{n} f\right\|_{p}^{p} \leq & \frac{p}{2 \pi} \int_{\mathbb{C}}|f(w)|^{p} e^{-\frac{p}{2}|w|^{2}} \\
& \times\left(\int_{\mathbb{C}} e^{\frac{p}{2}\left(\left|a^{n} z+\frac{b\left(1-a^{n}\right)}{1-a}\right|^{2}-|z|^{2}\right)}\left|u_{n}(z)\right|^{p} \chi_{D\left(\frac{w}{a^{n}}-\frac{b\left(1-a^{n}\right)}{1-a}, \frac{1}{|a|^{n}}\right)}(z) d A(z)\right) d A(w) .
\end{aligned}
$$

Using Lemma 2.5 and simplifying like the case for $p=\infty$, we get

$$
e^{\frac{p}{2}\left(\left|a^{n} z+\frac{b\left(1-a^{n}\right)}{1-a}\right|^{2}-|z|^{2}\right)}\left|u_{n}(z)\right|^{p} \leq e^{p c_{n}} e^{p\left|t_{n}\right||z|-p\left(\frac{1-|a|^{2 n}}{2}-\left|\frac{a_{2}\left(1-a^{2 n}\right)}{1-a^{2}}\right|\right)|z|^{2}}
$$

for all $z \in \mathbb{C}$. Since $W_{(u, \psi)}$ is compact and $\left|a_{2}\right|<\frac{1-|a|^{2}}{2}$, it follows that

$$
q_{n}-\left|p_{n}\right|=\frac{1-|a|^{2 n}}{2}-\left|\frac{a_{2}\left(1-a^{2 n}\right)}{1-a^{2}}\right|>0
$$

and

$$
\begin{gathered}
\int_{\mathbb{C}} e^{\frac{p}{2}\left(\left|a^{n} z+\frac{b\left(1-a^{n}\right)}{1-a}\right|^{2}-|z|^{2}\right)}\left|u_{n}(z)\right|^{p} \chi_{D\left(\frac{w}{a^{n}}-\frac{b\left(1-a^{n}\right)}{1-a}, \frac{1}{|a|^{n}}\right)}(z) d A(z) \\
\leq \int_{\mathbb{C}} e^{p c_{n}} e^{p\left|t_{n}\right||z|-p\left(\frac{1-|a|^{2 n}}{2}-\left|\frac{a_{2}\left(1-a^{2 n}\right)}{1-a^{2}}\right|\right)|z|^{2}} d A(z) \lesssim e^{p c_{n}}
\end{gathered}
$$

where we used the fact that $\sup _{n \in \mathbb{N}} t_{n}<\infty$ and $\inf _{n}\left\{q_{n}-\left|p_{n}\right|\right\}>0$. Hence,

$$
\left\|W_{(u, \psi)}^{n} f\right\|_{p} \lesssim e^{c_{n}}\|f\|_{p}
$$

and the conclusion follows as in the last part of $p=\infty$ and completes the proof of the theorem.

\section{The Spectrum of $W_{(u, \psi)}$}

In this section, we study the spectral of weighted compositions operators on all the Fock spaces $\mathcal{F}_{p}, 1 \leq p \leq \infty$. Let $T$ be a bounded linear operator on a Banach space 
$\mathcal{X}$. Then, the spectrum $\sigma(T)$ of $T$ is the set $\{\lambda \in \mathbb{C}: T-\lambda I$ is not invertible $\}$, where $I$ is the identity operator on $\mathcal{X}$. The spectrum $\sigma(T)$ is always a non-empty compact and closed subset of the disc centered at the origin and of radius $\|T\|$. It has been well known that the spectrum of an operator plays a vital role in the study of its dynamical properties; see [12]. Our next result will be used to prove mean ergodic results in the next section apart from being interest of its own.

Theorem 3.1 Let $1 \leq p \leq \infty, u, \psi \in \mathcal{H}(\mathbb{C})$ and $W_{(u, \psi)}$ be bounded on $\mathcal{F}_{p}$ and hence $\psi(z)=a z+b$ with $|a| \leq 1$. Then, if

(i) $W_{(u, \psi)}$ is compact and hence $|a|<1$, then

$$
\sigma\left(W_{(u, \psi)}\right)=\left\{0, u\left(\frac{b}{1-a}\right) a^{m}, \quad m \in \mathbb{N}_{0}\right\}
$$

(ii) $|a|=1$, then

$$
\sigma\left(W_{(u, \psi)}\right)=\left\{\begin{array}{l}
\overline{\left\{u(0) e^{\frac{a|b|^{2}}{a-1}} a^{m}: m \in \mathbb{N}_{0}\right\}}, \quad a \neq 1 \\
\left.z z:|z|=|u(0)| e^{\frac{|b|^{2}}{2}}\right\}, \quad a=1, \quad b \neq 0 \\
\{u(0)\}, \quad a=1, \quad b=0 .
\end{array}\right.
$$

We now remark a few points. First, observe that the number $b /(1-a)$ in (3.1) is the fixed point of the symbol $\psi$. We also note that when $a \neq 1$ and $|a|=1$, the expression in the spectrum can be expressed in terms of this fixed point. That is from Lemma 2.4, it follows that $u(0) e^{\frac{a|b|^{2}}{a-1}} a^{m}=u(b /(1-a)) a^{m}$. In this case, the spectrum contains finite number of points only when $a$ is a root of unity. Next, for $a \neq 1$, the necessity of the condition that $|u(b /(1-a))| \leq 1$ in Theorem 2.1 and Theorem 2.3 can be easily deduced using Theorem 3.1 since the spectrum of a power-bounded operator is always a subset of the closed unit disc. Here, for $|a|=1$ and $a \neq 1$, note that $\left|u(0) e^{\frac{a|b|^{2}}{a-1}}\right|=\left|u\left(\frac{b}{1-a}\right)\right|$. Another application of Theorem 3.1 will be given in Sect. 4. It is known that the spectrum plays an essential role in the study of theory of semigroups of linear operators [9] as well.

\subsection{Proof of Theorem 3.1}

(i). Let $W_{(u, \psi)}$ be compact and hence $|a|<1$. Here, our proof is based on an argument that goes back to [13]. We set $z_{0}=b /(1-a)$ and plan to show that the range of $W_{(u, \psi)}-a^{n} u\left(z_{0}\right) I$ fails to contain the complex polynomial $z^{n}$. Setting $n=1$ and arguing in the direction of contradiction, assume that there exists an $f \in \mathcal{F}_{p}$ such that

$$
u(z) f(\psi(z))-a u\left(z_{0}\right) f(z)=z .
$$

If $u\left(z_{0}\right)=0$ or $\mathrm{a}=0$, then $a u\left(z_{0}\right)=0$ and belongs to the spectrum. Thus, we may assume that $z_{0}$ is not in the zero set of $u$ and $a \neq 0$. First, assume that $z_{0}=0$. Then, 
taking $z=0$ in (3.2), we obtain that $f(0)=0$. On the other hand, differentiating both sides of Eq. (3.2) and setting again $z=0$, we obtain

$$
u^{\prime}(0) f(\psi(0))+u(0) \psi^{\prime}(0) f^{\prime}(\psi(0))-a u(0) f^{\prime}(0)=1
$$

which results in the contradiction $0=1$.

Similarly for $n>1$, differentiating both sides of the equation

$$
u(z) f(\psi(z))-a^{n} u\left(z_{0}\right) f(z)=z^{n}
$$

repeatedly and eventually setting $z=0$, we obtain $f^{(m)}(0)=0$ for all $m<n$, while for $m=n$ we get again the contradiction $0=n !$.

If $z_{0} \neq 0$, then we may set $\psi_{1}(z)=a z$,

$$
u_{1}(z)=\frac{u\left(z+z_{0}\right)}{\left\|K_{-z_{0}}\right\|_{2}^{2}} e^{-\overline{z_{0}} z+\overline{z_{0}}\left(a z+a z_{0}+b\right)}
$$

and observe that $\psi_{1}(0)=0$ and $u_{1}(0)=u\left(z_{0}\right)$. A straightforward calculation shows that

$$
W_{\left(u_{2}, \psi_{2}\right)} W_{(u, \psi)} W_{\left(u_{2}, \psi_{2}\right)}^{-1}=W_{\left(u_{1}, \psi_{1}\right)}
$$

and $W_{\left(u_{2}, \psi_{2}\right)}^{-1}=W_{\left(u_{3}, \psi_{3}\right)}$ where $u_{2}(z)=k_{-z_{0}}(z), \psi_{2}(z)=z+z_{0}, u_{3}=k_{z_{0}}$, and $\psi_{3}(z)=z-z_{0}$. It follows that the weighted composition operators $W_{\left(u_{1}, \psi_{1}\right)}$ and $W_{(u, \psi)}$ are similar and have the same spectrum, and our conclusion follows from the first case discussed above. Therefore, the set in the right-hand side of (3.1) in this case is contained in the spectrum.

Conversely, if $|a|<1$, then $W_{(u, \psi)}$ is compact and its spectrum contains only zero and eigenvalues. Thus, we consider a nonzero eigenvalue $\lambda \in \sigma\left(W_{(u, \psi)}\right.$ and show that it is of the form $u\left(z_{0}\right) a^{n}$ for some positive integer $n$. If $f$ is a corresponding nonzero eigenvector, then

$$
W_{(u, \psi)} f(z)=u(z) f(a z+b)=\lambda f(z)
$$

for all $z$ in $\mathbb{C}$. If $f$ has no zero at $z_{0}$, then (3.3) implies $u\left(z_{0}\right)=\lambda$ and hence $\lambda=$ $a^{0} u\left(z_{0}\right)$. On the other hand, if $f$ has zero at $z_{0}$ of order $m$, we may write

$$
f(z)=\left(z-z_{0}\right)^{m} g(z)
$$

where $g\left(z_{0}\right) \neq 0$. Then, substituting $f$ by this in (3.3) and differentiating both sides of the equation $m$ times and eventually setting $z=z_{0}$, we only get

$$
a^{m} u\left(z_{0}\right) m ! g\left(z_{0}\right)=\lambda m ! g\left(z_{0}\right)
$$

as all the other terms have factor $z-z_{0}$ and vanish. Now, $g\left(z_{0}\right)$ is nonzero and (3.4) holds only if $\lambda=a^{m} u\left(z_{0}\right)$ as asserted. 
The argument in the proof of part (ii) is divided into three cases depending on the values of $a$ and $b$.

Case 1. Let $|a|=1$ and $a \neq 1$. For simplicity, we first set $\psi_{z_{0}}(z)=z-z_{0}$ and claim that the weighted composition operator induced by $\left(k_{z_{0}}, \psi_{z_{0}}\right)$ is an isometric bijective map on $\mathcal{F}_{p}$ with inverse $W_{\left(k_{-z_{0}}, \psi_{z_{0}}^{-1}\right)}$. To this claim, for every $f \in \mathcal{F}_{p}$

$$
\begin{aligned}
\left\|W_{\left(k_{z_{0}}, \psi_{z_{0}}\right)} f\right\|_{p}^{p} & =\frac{p}{2 \pi} \int_{\mathbb{C}}\left|k_{z_{0}}(z)\right|^{p}\left|f\left(z-z_{0}\right)\right|^{p} e^{-\frac{p}{2}|z|^{2}} d A(z) \\
& =\frac{p}{2 \pi} \int_{\mathbb{C}}\left|f\left(z-z_{0}\right)\right|^{p} e^{-\frac{p}{2}\left|z-z_{0}\right|^{2}}\left(\left|k_{z_{0}}(z)\right|^{p} e^{\frac{p}{2}\left|z-z_{0}\right|^{2}-\frac{p}{2}|z|^{2}}\right) d A(z) \\
& =\frac{p}{2 \pi} \int_{\mathbb{C}}\left|f\left(z-z_{0}\right)\right|^{p} e^{-\frac{p}{2}\left|z-z_{0}\right|^{2}} d A(z)=\|f\|_{p}^{p}
\end{aligned}
$$

for all $1 \leq p<\infty$ which also holds true for $p=\infty$. This shows that the operator is a linear isometry and hence satisfies the injectivity condition $W_{\left(k_{z_{0}}, \psi_{z_{0}}\right)}^{-1} W_{\left(k_{z_{0}}, \psi_{z_{0}}\right)}=I$. On the other hand, for each $f \in \mathcal{F}_{p}$

$$
W_{\left(k_{z_{0}}, \psi_{z_{0}}\right)} W_{\left(k_{-z_{0}}, \psi_{z_{0}}^{-1}\right)} f(z)=k_{z_{0}}(z) k_{-z_{0}}\left(z-z_{0}\right) f(z)=f(z)
$$

which also shows that $W_{\left(k_{z_{0}}, \psi_{z_{0}}\right)} W_{\left(k_{z_{0}}, \psi_{z_{0}}\right)}^{-1}=I$, and hence the claim.

Next, using $z_{0}=b /(1-a)$ and Lemma 2.4 for every $f \in \mathcal{F}_{p}$ we compute

$$
\begin{aligned}
& W_{\left(k_{-z_{0}}, \psi_{z_{0}}^{-1}\right)} W_{(u, \psi)} W_{\left(k_{z_{0}}, \psi_{z_{0}}\right)} f(z) \\
& \left.\quad=k_{-z_{0}}(z) u\left(\psi_{z_{0}}^{-1}(z)\right)\right) k_{z_{0}}\left(\psi\left(\psi_{z_{0}}^{-1}(z)\right) f\left(\psi_{z_{0}}\left(\psi\left(\psi_{z_{0}}^{-1}(z)\right)\right)\right)\right. \\
& \quad=k_{-z_{0}}(z) u(0) K_{-\bar{a} b}\left(z+z_{0}\right) k_{z_{0}}\left(a z+b+a z_{0}\right) f(a z)=u(0) e^{\frac{|b|^{2}}{1-\bar{a}}} C_{\Psi_{0}} f(z)
\end{aligned}
$$

where $C_{\psi_{0}}$ is the composition operator induced by the symbol $\Psi_{0}(z)=a z$. This shows that $W_{(u, \psi)}$ is similar to the composition operator, up to a multiple, $C_{\Psi_{0}}$. Thus, $\sigma\left(W_{(u, \psi)}\right)=u(0) e^{\frac{|b|^{2}}{1-\bar{a}}} \sigma\left(C_{\Psi_{0}}\right)$. Using the spectrum of $C_{\Psi_{0}}$ from Theorem 2.6 of [23] and observing that $(1-\bar{a})^{-1}=a /(1-a)$ when $|a|=1$ and $a \neq 1$, we arrive at the desired conclusion.

Case 2. Let $a=1$ and $b \neq 0$. Applying Lemma 2.4,

$$
W_{u, \psi}=u \cdot C_{\psi}=u(0) K_{-b} C_{\psi}=u(0) e^{\frac{|b|^{2}}{2}} k_{-b} C_{\psi}=u(0) e^{\frac{|b|^{2}}{2}} W_{\left(k_{-b}, \psi\right)}
$$

The weighted composition operator $W_{\left(k_{-b}, \psi\right)}$ is unitary. Recall that the spectrum of a unitary operator lies on the unit circle $\mathbb{T}$. We claim that the spectrum of $W_{\left(k_{-b}, \psi\right)}$ is $\mathbb{T}$. To prove the claim, for any nonzero $w \in \mathbb{C}$ and $f \in \mathcal{F}_{p}$ we have 


$$
\begin{aligned}
& W_{\left(k_{-w}, \psi_{w}^{-1}\right)} W_{\left(k_{-b}, \psi\right)} W_{\left(k_{w}, \psi_{w}\right)} f(z) \\
& \quad=k_{-w}(z) k_{-b}(z+w) k_{w}(z+b+w) f(z+b) \\
& \quad=k_{-b}(z) e^{2 i \Im(\bar{w} b)} f(z+b)=e^{2 i \Im(\bar{w} b)} W_{\left(k_{-b}, \psi\right)} f(z)
\end{aligned}
$$

which shows that $W_{\left(k_{-b}, \psi\right)}$ is similar to $e^{2 i \Im(\bar{w} b)} W_{\left(k_{-b}, \psi\right)}$ for any $w \in \mathbb{C}$. Since $b \neq 0$, and $e^{2 i \Im(\bar{w} b)}$ is unimodular, and the spectrum of a unitary operator lies on the unit circle $\mathbb{T}$, it follows that the whole unit circle constitutes the spectrum.

Therefore, combining this with (3.5)

$$
\sigma\left(W_{(u, \psi)}\right)=u(0) e^{\frac{|b|^{2}}{2}} \sigma\left(W_{\left(k_{-b}, \psi\right)}\right)=u(0) e^{\frac{|b|^{2}}{2}} \mathbb{T}=\left\{z:|z|=|u(0)| e^{\frac{|b|^{2}}{2}}\right\} .
$$

and completes the proof.

Case 3. Let $a=1$ and $b=0$. In this case, the operator $W_{(u, \psi)}$ reduces to the multiplication operator $M_{u}$ where its spectrum has been already identified in Lemma 2.3 of [21].

\section{Uniformly Mean Ergodic $W_{(u, \psi)}$}

Having identified conditions under which $W_{(u, \psi)}$ is power bounded, we next turn our attention to the mean and uniformly mean ergodic properties of $W_{(u, \psi)}$ on $\mathcal{F}_{p}$. We may first state the following result about compact weighted composition operators on the spaces.

Proposition 4.1 Let $1 \leq p \leq \infty$ and $W_{(u, \psi)}$ be a compact power bounded operator on $\mathcal{F}_{p}$, and hence $\psi(z)=a z+b$ such that $|a|<1$. Let $u$ be non-vanishing on $\mathbb{C}$. Then, $W_{(u, \psi)}$ is uniformly mean ergodic.

Proof A result of Yosida and Kakutani ( [29] Theorem 4 and Corollary on page 204205 and Theorem 2.8 in [14]) implies that every compact power-bounded operator on a Banach space is uniformly mean ergodic. Thus, $W_{(u, \psi)}$ is uniformly mean ergodic.

Theorem 4.2 Let $1 \leq p<\infty$ and $W_{(u, \psi)}$ be a compact power bounded operator on $\mathcal{F}_{p}$, and hence, $\psi(z)=a z+b$ such that $|a|<1$. Let $u$ be non-vanishing on $\mathbb{C}$ such that $u\left(z_{0}\right)=1$ where $z_{0}=b /(1-a)$. Then,

$$
\lim _{n \rightarrow \infty}\left\|\frac{1}{n} \sum_{k=1}^{n} W_{(u, \psi)}^{k}-W_{\left(u_{\infty}, z_{0}\right)}\right\|=0, \quad u_{\infty}(z)=\prod_{j=0}^{\infty} u\left(\psi^{j}(z)\right) .
$$

Proof To prove (4.1), we argue as follows. First, observe that $u_{\infty}$ is a well-defined product as $W_{(u, \psi)}$ is power bounded; Lemma 2.6 and (1.2) imply

$$
\left|u_{\infty}(z)\right|=\lim _{n \rightarrow \infty}\left|u_{n}(z)\right| \lesssim \lim _{n \rightarrow \infty} e^{\frac{1}{2}|z|^{2}}\left\|u_{n}\right\|_{p} \lesssim e^{\frac{1}{2}|z|^{2}} .
$$


For each $f \in \mathcal{F}_{p}$, we claim that

$$
\lim _{n \rightarrow \infty}\left\|W_{(u, \psi)}^{n} f-W_{\left(u_{\infty}, z_{0}\right)} f\right\|_{p}=0 .
$$

Observe that (4.2) implies (4.1). Thus, we proceed to prove the claim by considering two different cases. Let $p<\infty$. Since $W_{(u, \psi)}$ is power bounded, by Lemma 2.6, $u_{n}$ belongs to $\mathcal{F}_{p}$ for all $n \in \mathbb{N}$. On the other hand, using the representation of $u_{n}$ in (2.17) and applying Theorem 2.3 and Lemma 2.7

$$
\left|u_{n}(z)\right| \lesssim e^{\left|t_{n} z\right|+\left|\frac{a_{2}\left(1-a^{2 n}\right)}{1-a^{2}}\right||z|^{2}} \leq e^{\left|t_{n} z\right|+\left|a_{2}\right| \frac{1-|a|^{2 n}}{1-|a|^{2}}|z|^{2}}
$$

where

$$
t_{n}=\frac{a_{1}\left(1-a^{n}\right)}{1-a}+\frac{2 a_{2} b}{1-a}\left(\frac{1-a^{n}}{1-a}-\frac{1-a^{2 n}}{1-a^{2}}\right)+\frac{\overline{\left(1-a^{n}\right) b}}{1-\bar{a}} a^{n},
$$

and letting $n \rightarrow \infty$

$$
\left|u_{\infty}(z)\right| \lesssim e^{\left(\frac{2\left|a_{1}\right|}{|1-a|}+\frac{2\left|a_{2} b\right|}{|1-a|}\left(\frac{2}{|1-a|}+\frac{2}{\left|1-a^{2}\right|}\right)\right)|z|+\frac{\left|a_{2}\right|}{\left|1-a^{2}\right|}|z|^{2}}
$$

The compactness condition $\left|a_{2}\right|<\frac{1-|a|^{2}}{2}$ implies $\frac{\left|a_{2}\right|}{\left|1-a^{2}\right|}<\frac{1}{2}$, which shows that $u_{\infty} \in \mathcal{F}_{p}$.

Moreover, by continuity,

$$
\lim _{n \rightarrow \infty}\left|W_{(u, \psi)}^{n} f(z)-W_{\left(u_{\infty}, z_{0}\right)} f(z)\right|=0,
$$

and since $W_{(u, \psi)}$ is power bounded, there is a constant $\alpha>0$ such that for every $n \in \mathbb{N}$

$$
\int_{\mathbb{C}}\left|W_{(u, \psi)}^{n} f(z)\right|^{p} e^{-\frac{p}{2}|z|^{2}} d A(z) \leq \int_{\mathbb{C}} \alpha^{p}|f(z)|^{p} e^{-\frac{p}{2}|z|^{2}} d A(z) .
$$

Consequently, we have

$$
\begin{aligned}
& \int_{\mathbb{C}}\left|W_{(u, \psi)}^{n} f(z)-W_{\left(u_{\infty}, z_{0}\right)} f(z)\right|^{p} e^{-\frac{p}{2}|z|^{2}} d A(z) \\
& \leq \int_{\mathbb{C}} 2^{p}\left(\alpha^{p}|f(z)|^{p}+\left|W_{\left(u_{\infty}, z_{0}\right)} f(z)\right|^{p}\right) e^{-\frac{p}{2}|z|^{2}} d A(z)<\infty .
\end{aligned}
$$

Applying Lebesgue dominated convergent theorem on the sequence

$$
g_{n}(z):=\left|W_{(u, \psi)}^{n} f(z)-W_{\left(u_{\infty}, z_{0}\right)} f(z)\right|^{p} e^{-\frac{p}{2}|z|^{2}},
$$


we get that

$$
\lim _{n \rightarrow \infty}\left\|W_{(u, \psi)}^{n} f-W_{\left(u_{\infty}, z_{0}\right.} f\right\|_{p}^{p}=\frac{p}{2 \pi} \lim _{n \rightarrow \infty} \int_{\mathbb{C}} g_{n}(z) d A(z)=\frac{p}{2 \pi} \int_{\mathbb{C}} \lim _{n \rightarrow \infty} g_{n}(z) d A(z)=0
$$

as claimed.

Theorem 4.3 Let $W_{(u, \psi)}$ be a compact power bounded operator on $\mathcal{F}_{\infty}$, and hence, $\psi(z)=a z+b$ such that $|a|<1$. Let $u$ be non-vanishing on $\mathbb{C}$ such that $u\left(z_{0}\right)=1$, where $z_{0}=b /(1-a)$. Then,

$$
\lim _{n \rightarrow \infty}\left\|\frac{1}{n} \sum_{k=1}^{n} W_{(u, \psi)}^{k}-W_{\left(u_{\infty}, z_{0}\right)}\right\|=0, u_{\infty}(z)=\prod_{j=0}^{\infty} u\left(\psi^{j}(z)\right)
$$

Proof For this, we consider the subspace $\mathcal{F}_{0}$ of $\mathcal{F}_{\infty}$ defined by

$$
\mathcal{F}_{0}=\left\{f \in \mathcal{F}_{\infty}: \lim _{|z| \rightarrow \infty}|f(z)| e^{-\frac{1}{2}|z|^{2}}=0\right\}
$$

This subspace is closed in $\mathcal{F}_{\infty}$, and it contains polynomials. Moreover, the polynomials are dense in $\mathcal{F}_{0}$, and $\mathcal{F}_{\infty}$ is canonically isomorphic to the bidual of $\mathcal{F}_{0}$; see for details in [4]. We proceed to show first that (4.2) holds for each $f \in \mathcal{F}_{0}$. It is easy to see that as $n \rightarrow \infty$

$$
\psi^{n}(z)=a^{n} z+z_{0}\left(1-a^{n}\right) \rightarrow z_{0}
$$

uniformly on compact subsets of $\mathbb{C}$.

Next, we show that $u_{n} \rightarrow u_{\infty}$ uniformly on compact subset of $\mathbb{C}$ also. Since $u_{\infty} \in \mathcal{F}_{1}$

$$
\int_{\mathbb{C}}\left|u_{n}(z)-u_{\infty}(z)\right| e^{-\frac{1}{2}|z|^{2}} d A(z) \leq \int_{\mathbb{C}} 2\left(\alpha+\left|u_{\infty}(z)\right|\right) e^{-\frac{1}{2}|z|^{2}} d A(z)<\infty .
$$

Applying Lebesgue dominated convergence theorem, for $z \in K$, where $K$ is compact subset of $\mathbb{C}$,

$$
\begin{aligned}
\lim _{n \rightarrow \infty}\left|u_{n}(z)-u_{\infty}(z)\right| & \leq \lim _{n \rightarrow \infty} e^{\frac{1}{2}|z|^{2}}\left\|u_{n}-u_{\infty}\right\|_{1} \leq\left(\max _{z \in K} e^{\frac{1}{2}|z|^{2}}\right) \lim _{n \rightarrow \infty}\left\|u_{n}-u_{\infty}\right\|_{1} \\
& =\left(\max _{z \in K} e^{\frac{1}{2}|z|^{2}}\right) \lim _{n \rightarrow \infty} \int_{\mathbb{C}}\left|u_{n}(w)-u_{\infty}(w)\right| e^{-\frac{1}{2}|w|^{2}} d A(w)=0 .
\end{aligned}
$$

From this, we have

$$
u_{n}(z) f\left(\psi^{n}(z)\right) \rightarrow u_{\infty}(z) f\left(z_{0}\right)
$$


uniformly on the compact subsets of $\mathbb{C}$. That is, for each compact set $K$ in $\mathbb{C}$,

$$
\sup _{z \in K}\left|u_{n}(z) f\left(\psi^{n}(z)\right)-u_{\infty}(z) f\left(z_{0}\right)\right| \rightarrow 0
$$

as $n \rightarrow \infty$.

Next, with $f \in \mathcal{F}_{0}$ and each $n$,

$$
\begin{aligned}
\lim _{|z| \rightarrow \infty}\left|W_{(u, \psi)}^{n} f(z)\right| e^{-\frac{1}{2}|z|^{2}} & =\lim _{|z| \rightarrow \infty}\left|u_{n}(z)\right|\left|f\left(a^{n} z+z_{0}\left(1-a^{n}\right)\right)\right|^{2} e^{-\frac{1}{2}|z|^{2}} \\
\leq & \sup _{z \in \mathbb{C}}\left(\left|u_{n}(z)\right| e^{\frac{1}{2}\left(\left|a^{n} z+z_{0}\left(1-a^{n}\right)\right|^{2}-|z|^{2}\right)}\right) \\
& \times \lim _{|z| \rightarrow \infty}\left|f\left(a^{n} z+v\right)\right|^{2} e^{-\frac{1}{2}\left|a^{n} z+z_{0}\left(1-a^{n}\right)\right|^{2}} \\
& \lesssim \lim _{|z| \rightarrow \infty}\left|f\left(a^{n} z+z_{0}\left(1-a^{n}\right)\right)\right|^{2} e^{-\frac{1}{2}\left|a^{n} z+v\right|^{2}}=0 .
\end{aligned}
$$

Note that the last inequality above holds since $W_{(u, \psi)}$ is power bounded and the supremum above is uniformly bounded. That is,

$$
\sup _{n} \sup _{z \in \mathbb{C}}\left(\left|u_{n}(z)\right| e^{\frac{1}{2}\left(\left|a^{n} z+z_{0}\left(1-a^{n}\right)\right|^{2}-|z|^{2}\right)}\right) \lesssim\left|u\left(z_{0}\right)\right|^{n} \leq 1 .
$$

Furthermore, $u_{\infty} \in \mathcal{F}_{0}$ since it belongs to $\mathcal{F}_{p}$ for all $p \leq \infty$.

Now given $\varepsilon>0, f \in \mathcal{F}_{0}$ and since $u_{\infty} \in \mathcal{F}_{0}$, we can find $r_{0}>0$ such that $\left|W_{(u, \psi)}^{n} f(z)\right| e^{-\frac{1}{2}|z|^{2}}<\varepsilon / 2$ and $\left|u_{\infty}(z)\right|\left|f\left(z_{0}\right)\right| e^{-\frac{1}{2}|z|^{2}}<\varepsilon / 2$ whenever $|z|>r_{0}$. Then, for each $|z|>r_{0}$ and $n \in \mathbb{N}$, we have

$$
\begin{aligned}
\left|W_{(u, \psi)}^{n} f(z)-u_{\infty}(z) f\left(z_{0}\right)\right| e^{-\frac{1}{2}|z|^{2}} \leq & \left|W_{(u, \psi)}^{n} f(z)\right| e^{-\frac{1}{2}|z|^{2}} \\
& +\left|u_{\infty}(z) f\left(z_{0}\right)\right| e^{-\frac{1}{2}|z|^{2}}<\varepsilon .
\end{aligned}
$$

We apply (4.5) to the compact set $K_{0}=\left\{z \in \mathbb{C}:|z| \leq r_{0}\right\}$ to find $n_{0}$ such that if $z \in K_{0}$ and $n \geq n_{0}$, we have

$$
\left|u_{n}(z) f\left(\psi^{n}(z)\right)-u_{\infty}(z) f\left(z_{0}\right)\right|<\frac{\varepsilon}{2 S}
$$

with $S:=\max _{z \in K_{0}} e^{-\frac{1}{2}|z|^{2}}$. If $n \geq n_{0}$ and $z \in \mathbb{C}$, we have

$$
\left|W_{(u, \psi)}^{n} f(z)-u_{\infty}(z) f\left(z_{0}\right)\right| e^{-\frac{1}{2}|z|^{2}}<\varepsilon .
$$


Thus,

$$
\lim _{n \rightarrow \infty}\left\|W_{(u, \psi)}^{n} f-u_{\infty} f\left(z_{0}\right)\right\|_{\infty}=0 .
$$

Next, we show (4.3) for $p=\infty$. Since $\mathcal{F}_{\infty}$ is canonically isomorphic to the bidual of $\mathcal{F}_{0}$, and the bitranspose operator $W_{(u . \psi)}^{\prime \prime}$ of $W_{(u, \psi)}: \mathcal{F}_{0} \rightarrow \mathcal{F}_{0}$ coincides with weighted composition operator $W_{(u, \psi)}: \mathcal{F}_{\infty} \rightarrow \mathcal{F}_{\infty}$, the conclusion follows from the well-known fact that $\|T\|=\left\|T^{\prime}\right\|=\left\|T^{\prime \prime}\right\|$ for any bounded operator $T$ on a Banach space.

The preceding results assure that $W_{(u, \psi)}$ with $\psi(z)=a z+b,|a|<1$ is always uniformly mean ergodic whenever it is compact and power bounded. Now, we consider the case when $\psi(z)=a z+b$ and $|a|=1$. Note that power boundedness in this case implies that either $|u(0)|=e^{-\frac{|b|^{2}}{2}}$ or $|u(0)|<e^{-\frac{|b|^{2}}{2}}$. In 1939, Lorch [19] proved that every power-bounded operator on a reflexive Banach space is mean ergodic. The same result was later obtained in reflexive Frechet spaces [1]. Accordingly, as the spaces $\mathcal{F}_{p}$ are reflexive for all $1<p<\infty$, every power-bounded $W_{(u, \psi)}$ is mean ergodic. Thus, for such spaces we will consider conditions under which the ergodicity becomes uniform.

Theorem 4.4 (i) Let $1 \leq p \leq \infty, \psi(z)=a z+b$ with $|a|=1$ and $|u(0)|<e^{-\frac{|b|^{2}}{2}}$. Then, $W_{(u, \psi)}$ is uniformly mean ergodic on $\mathcal{F}_{p}$, and

$$
\lim _{n \rightarrow \infty}\left\|\frac{1}{n} \sum_{k=0}^{n} W_{(u, \psi)}^{k}\right\|=0 .
$$

(ii) Let $1 \leq p \leq \infty$, and $\psi(z)=$ az with $|a|=1$. If both $u(0)$ and a are roots of unity, then $W_{(u, \psi)}$ is uniformly mean ergodic on $\mathcal{F}_{p}$.

By a result in [26], the composition operator $C_{\psi}$ is not uniformly mean ergodic on $\mathcal{F}_{\infty}$ whenever $|a|=1$. Now the weight function $u$ makes it possible to enrich uniformity by taking the value $|u(0)|$ smaller.

Proof (i) Applying the assumption along with (2.13)

$$
\begin{aligned}
\lim _{n \rightarrow \infty}\left\|\frac{1}{n} \sum_{k=0}^{n} W_{(u, \psi)}^{k}\right\| & \leq \lim _{n \rightarrow \infty} \frac{1}{n} \sum_{k=0}^{n}\left\|W_{(u, \psi)}^{k}\right\| \lesssim \lim _{n \rightarrow \infty} \frac{1}{n} \sum_{k=0}^{n}\left(|u(0)| e^{\frac{|b|^{2}}{2}}\right)^{k} \\
& \leq \lim _{n \rightarrow \infty} \frac{2 n^{-1}}{1-|u(0)| e^{\frac{|b|^{2}}{2}}}=0
\end{aligned}
$$

as claimed.

(ii) By assumption, there exist numbers $m, N \in \mathbb{N}$ such that $a^{N}=1=u(0)^{m}$. Consider the smallest positive integer $N_{0} \leq m N$ such that $a^{N_{0}}=u(0)^{N_{0}}=1$. In 
this case, the sequence $W_{u, \psi}^{n}$ is periodic with period $N_{0}$. Any $n \in \mathbb{N}$ can be written in the form of $n=N_{0} l+j$ for some $l \in \mathbb{N}$ and $j=0,1,2, \ldots, N_{0}-1$. Thus,

$$
\begin{aligned}
& \lim _{n \rightarrow \infty}\left\|\frac{1}{n} \sum_{k=1}^{n} W_{(u, \psi)}^{k}-\frac{1}{N_{0}} \sum_{k=1}^{N_{0}} W_{(u, \psi)}^{k}\right\| \\
& =\lim _{l \rightarrow \infty} \frac{1}{\left(N_{0} l+j\right)}\left\|\sum_{k=1}^{j} W_{(u, \psi)}^{k}-\frac{j}{N_{0}} \sum_{k=1}^{N_{0}} W_{(u, \psi)}^{k}\right\| \\
& \leq \lim _{l \rightarrow \infty} \frac{1}{\left(N_{0} l+j\right)}\left(\sum_{k=1}^{j}\left\|W_{(u, \psi)}^{k}\right\|+\frac{j}{N_{0}} \sum_{k=1}^{N_{0}}\left\|W_{(u, \psi)}^{k}\right\|\right)=0
\end{aligned}
$$

and completes the proof.

Next, we consider the cases when the uniform ergodicity fails. We may first recall that a Banach space $X$ is a Grothendieck space if every sequence $\left(x_{n}\right)$ in $X^{\prime}$ which is convergent to 0 for the weak topology $\sigma\left(X^{\prime}, X\right)$ is also convergent to 0 for the weak topology $\sigma\left(X^{\prime}, X^{\prime \prime}\right)$. The space $X$ has the Dunford-Pettis property if for any sequence $\left(x_{n}\right)$ in $X$ which is convergent to 0 for the weak topology $\sigma\left(X, X^{\prime}\right)$ and any sequence $\left(x_{n}^{\prime}\right)$ in $X^{\prime}$ which is convergent to 0 for the weak topology $\sigma\left(X^{\prime}, X^{\prime \prime}\right)$ one gets $\lim _{n \rightarrow \infty} x_{n}^{\prime}\left(x_{n}\right)=0$. The spaces $\ell^{\infty}$ or $H^{\infty}(\mathbb{D})$ are examples of Grothendieck spaces with Dunford-Pettis property [17].

Theorem 4.5 Let $1 \leq p \leq \infty$ and $W_{(u, \psi)}$ is power bounded on $\mathcal{F}_{p}$ with $\psi(z)=a z$, $|a|=1$. Then, $W_{(u, \psi)}$ is

(i) mean ergodic on $\mathcal{F}_{p}$ for all $1 \leq p<\infty$ if $1 \neq u(0) a^{m}$ for all $m \in \mathbb{N}_{0}$, and for each $f \in \mathcal{F}_{p}$

$$
\lim _{n \rightarrow \infty}\left\|\frac{1}{n} \sum_{k=1}^{n} W_{(u, \psi)}^{k} f\right\|_{p}=0,
$$

(ii) mean ergodic on $\mathcal{F}_{p}$ for all $1 \leq p<\infty$ if $u(0)=1$ and a is not root of unity, and for each $f \in \mathcal{F}_{p}$

$$
\lim _{n \rightarrow \infty}\left\|\frac{1}{n} \sum_{k=1}^{n} W_{(u, \psi)}^{k} f-f(0)\right\|_{p}=0,
$$

(iii) not uniformly mean ergodic on $\mathcal{F}_{p}$ for all $1 \leq p \leq \infty$, and not mean ergodic on $\mathcal{F}_{\infty}$ whenever $1 \neq u(0) a^{m}$ for all $m \in \mathbb{N}_{0}$.

Proof (i) and (ii). We first check when $f$ belongs to the set of monomials. If $f=1$, then the result holds trivially. Thus, for $z^{m}, m \geq 1$. Assume that $1 \neq u(0) a^{m}$ for all 
$m \in \mathbb{N}$.

$$
\left\|\frac{1}{n} \sum_{k=1}^{n} W_{(u, \psi)}^{k} z^{m}\right\|_{p}=\left\|\frac{1}{n} \sum_{k=1}^{n} a^{m k} u_{k} z^{m}\right\|_{p} .
$$

Since $a^{k} u(0) \neq 1$ for each $k \in \mathbb{N}$ and $u_{k}(z)=u(0)^{k}$, or $u(0)=1$ and $a$ is not root of unity

$$
\begin{aligned}
\left\|\frac{1}{n} \sum_{k=1}^{n} a^{m k} u_{k} z^{m}\right\|_{p} & =\left\|\frac{1}{n} \sum_{k=1}^{n} u(0)^{k} a^{m k} z^{m}\right\|_{p}=\left\|\frac{z^{m}}{n} \frac{a^{m}\left(1-u(0)^{n} a^{m n}\right)}{1-a^{m} u(0)}\right\|_{p} \\
& \leq \frac{2\left\|z^{m}\right\|_{p}}{n\left|1-a^{m} u(0)\right|} \rightarrow 0
\end{aligned}
$$

as $n \rightarrow \infty$. Since the set of polynomials is dense in $\mathcal{F}_{p}$ and $W_{(u, \psi)}$ is power bounded on $\mathcal{F}_{p}$ by Theorem 2.1, we have the result (see, e.g., Lemma 2.1 in [3]).

(iii) Assume now on the contrary that $W_{u, \psi}$ is uniformly mean ergodic. Then, by the classical result of Lin [16]), $\operatorname{Im}\left(I-W_{(u, \psi)}\right)$ is closed, where $\operatorname{Im}\left(I-W_{(u, \psi)}\right)$ denotes the range of $I-W_{(u, \psi)}$. Moreover, by a result of Yosida [28] it follows that $\operatorname{Ker} T_{0}=\overline{\operatorname{Im}\left(I-W_{(u, \psi)}\right)}$, where $T_{0}=\lim _{n} T_{n}$ and $T_{n}=\frac{1}{n} \sum_{k=1}^{n} W_{(u, \psi)}^{k}$. Hence,

$$
\begin{aligned}
\operatorname{Im}\left(I-W_{(u, \psi)}\right) & =\overline{\operatorname{Im}\left(I-W_{(u, \psi)}\right)}=K \operatorname{er} T_{0}=\left\{f \in \mathcal{F}_{p}: \lim _{n \rightarrow \infty} \frac{1}{n} \sum_{k=1}^{n} W_{(u, \psi))}^{k} f=0\right\} \\
& =\left\{f \in \mathcal{F}_{p}: \lim _{n \rightarrow \infty} \frac{1}{n} \sum_{k=1}^{n} u(0)^{k} f\left(\psi^{k}\right)=0\right\},
\end{aligned}
$$

where the last equality follows after an application of Lemma 2.4. Furthermore,

$$
\mathcal{F}_{p}=\operatorname{Im}\left(I-W_{(u, \psi)}\right) \bigoplus \operatorname{Ker}\left(I-W_{(u, \psi)}\right) .
$$

We claim that $\operatorname{Ker}\left(I-W_{(u, \psi)}\right)$ contains only the constant functions. Indeed, if $f$ belongs to it, then since $u(0) f(a z)=f(z)$ for each $z \in \mathbb{C}$, we have $u(0) a^{n} f^{(n)}(a z)=$ $f^{(n)}(z)$, and hence $u(0) a^{n} f^{(n)}(0)=f^{(n)}(0)$. This yields $f^{(n)}(0)=0$ for every $n \in \mathbb{N}$ since $u(0) a^{n} \neq 1$. If we define $g(z)=f(z)-f(0)$, it follows that $g^{(n)}(0)=0$ for every $n=0,1,2, \ldots$ Hence $g(z)=0$ for every $z \in \mathbb{C}$. Therefore, $f(z)=f(0)$ for every $z \in \mathbb{C}$.

Next, we show that the constant functions belong to the set in (4.6) and contradicts (4.7). Thus, if $h=\alpha$ is a nonzero constant function, then $W_{(u, \psi))}^{k} h(z)=$ $u(0)^{k} h\left(\psi^{k}(z)\right)=u(0)^{k} \alpha$ for every $z \in \mathbb{C}$. Thus,

$$
\begin{aligned}
\left\|\frac{1}{n} \sum_{k=1}^{n} h\left(\psi^{k}\right)\right\|_{p} & \leq \frac{\|\alpha\|_{p}}{n}\left|\sum_{k=1}^{n} u(0)^{k}\right|=\frac{\|\alpha\|_{p}}{n}\left|\frac{u(0)-u(0)^{n+1}}{1-u(0)}\right| \\
& \leq \frac{2\|\alpha\|_{p}}{n|1-u(0)|} \rightarrow 0 \text { as } n \rightarrow \infty
\end{aligned}
$$


The case when $1=u(0)$ and $a$ is not a root of unity follows as a special case from Corollary 4.6.

It remains to show that $W_{(u, \psi)}$ is not mean ergodic on $\mathcal{F}_{\infty}$. Theorem 1.1 in [20] implies that $\mathcal{F}_{\infty}$ is isomorphic to $\ell^{\infty}$ or $H^{\infty}(\mathbb{D})$. Hence, $\mathcal{F}_{\infty}$ is Grothendieck spaces with Dunford-Pettis property. On the other hand, by a result of Lotz [18] every powerbounded mean ergodic operator on a Grothendieck Banach space with the DunfordPettis property is uniformly mean ergodic. Therefore, $W_{(u, \psi)}$ is not mean ergodic on $\mathcal{F}_{\infty}$

We remark that when $|a|=1$, the operators are isometric bijective with $W_{(u, \psi)}^{-1}=$ $W_{\left(v, \psi^{-1}\right)}$ where $v(z):=\overline{u(0)} K_{b}(z)$. This can be seen as for every $f \in \mathcal{F}_{p}$

$$
\begin{aligned}
\left\|W_{(u, \psi)} f\right\|_{p}^{p} & =\frac{p}{2 \pi}|u(0)|^{p} \int_{\mathbb{C}}\left|K_{-\bar{a} b}(z)\right|^{p}|f(a z+b)|^{p} e^{-\frac{p}{2}|z|^{2}} d A(z) \\
& =\frac{p}{2 \pi}|u(0)|^{p} \int_{\mathbb{C}}|f(a z+b)|^{p} e^{-\frac{p}{2}|a z+b|^{2}}\left(\left|K_{-\bar{a} b}(z)\right|^{p} e^{\frac{p}{2}|a z+b|^{2}-\frac{p}{2}|z|^{2}}\right) d A(z) \\
& =\frac{1}{|a|^{2}}|u(0)|^{p} e^{\frac{p|b|^{2}}{2}}\|f\|_{p}^{p}=\|f\|_{p}^{p}
\end{aligned}
$$

for all $1 \leq p<\infty$ which also holds true for $p=\infty$. This shows that the operator is a linear isometry and hence satisfies the injectivity condition $W_{(u, \psi)}^{-1} W_{(u, \psi)}=I$. On the other hand, for each $f \in \mathcal{F}_{p}$

$$
\begin{aligned}
W_{(u, \psi)} W_{\left(v, \psi^{-1}\right)} f(z) & =u(z) \overline{u(0)} K_{b}(\psi(z)) \cdot f\left(\psi^{-1}(\psi(z))\right) \\
& =u(z) \overline{u(0)} K_{b}(a z+b) f(z)=|u(0)|^{2} K_{-\bar{a} b}(z) K_{b}(a z+b) f(z)=f(z)
\end{aligned}
$$

which also shows that $W_{(u, \psi)} W_{(u, \psi)}^{-1}=I$. As shown below and Theorem 3.1, the spectrum of some of these class of operators is contained in the unit circle.

The uniformly mean ergodic results in part (ii) of Theorems 4.4 and 4.5 deal with when $\psi(z)=a z+b$ form with $|a|=1$ and $b=0$. The case for $b \neq 0$ is our next point of interest.

Corollary 4.6 Let $1 \leq p \leq \infty, \psi(z)=a z+b,|a|=1$ and $a$ is not a root of unity, $u\left(\frac{b}{1-a}\right)=1$ and hence $|u(0)|=e^{-\frac{|b|^{2}}{2}}$. Then, $W_{(u, \psi)}$ cannot be uniformly mean ergodic on $\mathcal{F}_{p}$.

Proof First, observe that in this case Theorem 3.1 and since for $|a|=1$ and $a \neq 1$,

$$
\left|u(0) e^{\frac{a\left|b^{2}\right|}{a-1}} a^{m}\right|=|u(0)| e^{|b|^{2} \Re\left(\frac{a}{a-1}\right)}=|u(0)| e^{|b|^{2} \Re\left(\frac{a(\bar{a}-1)}{(a-1)(\bar{a}-1)}\right)}=|u(0)| e^{\frac{\left|b^{2}\right|}{2}}=1,
$$

the spectrum $\sigma\left(W_{(u, \psi)}\right)$ is contained in the unit circle $\mathbb{T}$. Furthermore, as $a$ is not a root of unity, it follows that 1 is an accumulation point of the spectrum of $W_{(u, \psi)}$. Moreover, 1 is in the spectrum of $W_{(u, \psi)}$ since $u(0) e^{\frac{a\left|b^{2}\right|}{a-1}} a^{0}=u\left(\frac{b}{1-a}\right) a^{0}=1$. Then, an application of Theorem 3.16 of [8] gives the conclusion. 


\subsection{The Multiplication Operator}

We now conclude the section by specializing the main results made in the above section to the multiplication operator $M_{u}$ acting on Fock spaces. Note that from Lemma 2.3 in [21], it is known that the operator $M_{u}$ is bounded on $\mathcal{F}_{p}$ if and only if $u$ is a constant function. The same conclusion can be also easily drawn by applying the condition in (2.5) along with Liouville's theorem.

Corollary 4.7 Let $1 \leq p \leq \infty$ and $u \in \mathcal{H}(\mathbb{C})$ such that $M_{u}$ is bounded on $\mathcal{F}_{p}$. Then, the following statements are equivalent.

(i) $M_{u}$ is power bounded on $\mathcal{F}_{p}$;

(ii) $|u(0)| \leq 1$;

(iii) $M_{u}$ is mean ergodic on $\mathcal{F}_{p}$;

(iv) $M_{u}$ is uniformly mean ergodic on $\mathcal{F}_{p}$.

Proof The equivalency of (i) and (ii) is an immediate deduction from Theorem 2.1. Thus, we shall show (ii) $\Rightarrow$ (iii), (iii) $\Rightarrow$ (iv), and (iv) $\Rightarrow$ (i). For the first, simplifying the proof of Theorem 4.4 part (i) for the case $b=0$ and $a=1$, we get $W_{(u, \psi)}^{k}=M_{u}^{k}$ and

$$
\left\|\frac{1}{n} \sum_{k=1}^{n} M_{u}^{k} f\right\|_{p}=\left\|\frac{f}{n} \sum_{k=1}^{n} u_{k}(0)\right\|_{p}=\left\|\frac{f}{n} \sum_{k=1}^{n} u(0)^{k}\right\|_{p}=\|f\|_{p}\left|\frac{1}{n} \sum_{k=1}^{n} u(0)^{k}\right| .
$$

Consider first the case when $u(0) \neq 1$ and $|u(0)| \leq 1$. Then,

$$
\frac{\|f\|_{p}}{n}\left|\sum_{k=1}^{n} u(0)^{k}\right|=\frac{\|f\|_{p}}{n} \frac{|u(0)|\left|1-u(0)^{n}\right|}{|1-u(0)|} \leq \frac{2\|f\|_{p}}{n|1-u(0)|} \rightarrow 0
$$

as $n \rightarrow \infty$. Thus, $\frac{1}{n} \sum_{k=1}^{n} M_{u}^{k}$ converges pointwise to zero. If $u(0)=1$, then $M_{u}$ reduces to the identity map and the assertion follows trivially.

Next, we show that (iii) implies (iv). That is, the above convergence is uniform on the operator norm. Now the assumption implies that $\frac{M_{u}^{n} f}{n} \rightarrow 0$ as $n \rightarrow \infty$ for all $f \in \mathcal{F}_{p}$. In particular for $f=\mathbf{1}$, the statement $\frac{M_{u}^{n} \mathbf{1}}{n}=\frac{u(0)^{n}}{n} \rightarrow 0$ holds only if $|u(0)| \leq 1$. Now, for $u(0)=1$, the operator reduces again to the identity map. Thus, we consider the case when $u(0) \neq 1$ and argue

$$
\begin{aligned}
\left\|\frac{1}{n} \sum_{k=1}^{n} M_{u}^{k}\right\| & =\sup _{\|f\|_{p}=1}\left\|\frac{1}{n} \sum_{k=1}^{n} M_{u}^{k} f\right\|_{p}=\sup _{\|f\|_{p}=1}\left\|\frac{1}{n} \sum_{k=1}^{n} u(0)^{k} f\right\|_{p} \\
& \leq \frac{1}{n} \sum_{k=1}^{n}|u(0)|^{k}=\frac{\left.|u(0)||1-| u(0)\right|^{n} \mid}{n(1-|u(0)|)} \leq \frac{2}{n|1-u(0)|} \rightarrow 0, n \rightarrow \infty .
\end{aligned}
$$


Now assume that (iv) holds. Then, $\left\|M_{u}^{n}\right\| / n \rightarrow 0$ as $n \rightarrow \infty$. On the other hand, from (2.13) we get $\left\|M_{u}^{n}\right\|=|u(0)|^{n}$, which implies that $\left\|M_{u}^{n}\right\| / n \rightarrow 0$ only when $|u(0)| \leq 1$. Therefore, by Theorem 2.1 , the operator is power bounded.

Acknowledgements The authors would like to thank Professor José Bonet for useful discussions on the subject. We would also like to thank the anonymous reviewers for useful comments that helped us improve the manuscript.

Funding Open access funding provided by Western Norway University Of Applied Sciences.

Open Access This article is licensed under a Creative Commons Attribution 4.0 International License, which permits use, sharing, adaptation, distribution and reproduction in any medium or format, as long as you give appropriate credit to the original author(s) and the source, provide a link to the Creative Commons licence, and indicate if changes were made. The images or other third party material in this article are included in the article's Creative Commons licence, unless indicated otherwise in a credit line to the material. If material is not included in the article's Creative Commons licence and your intended use is not permitted by statutory regulation or exceeds the permitted use, you will need to obtain permission directly from the copyright holder. To view a copy of this licence, visit http://creativecommons.org/licenses/by/4.0/.

\section{References}

1. Albanese, A.A., Bonet, J., Ricker, W.J.: Mean ergodic operators in Fréchet spaces. Anal. Acad. Sci. Fenn. Math. 34, 401-436 (2009)

2. Beltrán-Meneu, M.J., Gómez-Collado, M.C., Jordá, E., Jornet, D.: Mean ergodicity of weighted composition operators on spaces of holomorphic functions. J. Math. Anal. Appl. 444(2), 1640-1651 (2016)

3. Beltrán-Meneu, M.J., Gómez-Collado, M.C., Jordá, E., Jornet, D.: Mean ergodic composition operators on Banach spaces of holomorphic functions. J. Funct. Anal. 270, 4369-4385 (2016)

4. Bierstedt, K.D., Summers, W.H.: Biduals of weighted Banach spaces of analytic functions. J. Austral. Math. Soc. Ser. 54, 70-79 (1993)

5. Bonet, J., Domański, P.: A note on mean ergodic composition operators on spaces of holomorphic functions,. Rev. R. Acad. Cienc. Exactas Fís. Nat. Ser. A Mat. RACSAM 105, 389-396 (2011)

6. Bonet, J., Ricker, W.J.: Mean ergodicity of multiplication operators in weighted spaces of holomorphic functions. Arch. Math. 92, 428-437 (2009)

7. Carroll, T., Gilmore, C.: Weighted composition operators on Fock Spaces and their dynamics. J. Math. Anal. Appl. https://doi.org/10.1016/j.jmaa.2021.125234

8. Dunford, N.: Spectral theory. I. Convergence to projections. Trans. Am. Math. Soc. 54, 185-217 (1943)

9. Engel, K.J., Nagel, R.: One-Parameter Semigroups for Linear Evolution Equations. Springer, New York (1999)

10. Fleming, R.J., Jamison, J.E.: Isometries on Banach Spaces: Function Spaces. Chapman and Hall, New York (2002)

11. Fleming, R.J., Jamison, J.E.: Isometries on Banach Spaces: Vector-Valued Function Spaces, vol. 2. Chapman and Hall/CRC (2008)

12. Grosse-Erdmann, K.G., Manguillot, A.P.: Linear Chaos. Springer, New York (2011)

13. Kamowitz, H.: The spectra of endomorphisms of the disc algebra. Pac. J. Math. 46, 433-440 (1973)

14. Krengel, U.: Ergodic Theorems. Walter de Gruyter, Berlin (1985)

15. Le, T.: Normal and isometric weighted composition operators on the Fock space. Bull. Londo. Math. Soc. 46, 847-856 (2014)

16. Lin, M.: On the uniform ergodic theorem. Proc. Am. Math. Soc 43(2), 337-340 (1974)

17. Lotz, H. P.: Tauberian theorems for operators on L1 and similar spaces: Functional Analysis: Surveys and Recent Results III, K.D. Bierstedt and b. Fuchssteiner (Eds.), North Holland, Amsterdam, 117-133 (1984)

18. Lotz, H.P.: Uniform convergence of operators on $L^{\infty}$ and similar spaces. Math. Z. 190, 207-220 (1985)

19. Lorch, E.R.: Means of iterated transformations in reflexive vector spaces. Bull. Am. Math. Soc. 45, 945-957 (1939) 
20. Lusky, W.: On the isomorphism classes of weighted spaces of harmonic and holomorphic functions. Studia Math. 175, 19-45 (2006)

21. Mengestie, T.: Spectral properties of Volterra-type integral operators on Fock-Sobolev spaces. J. Korean Math. Soc. 54(6), 1801-1816 (2017)

22. Mengestie, T., Worku, M.: Topological structures of generalized Volterra-type integral operators. Mediterr. J. Math. 15, 42 (2018). https://doi.org/10.1007/s00009-018-1080-5

23. Mengestie, T., Seyoum, W.: Spectral properties of composition operators on Fock-type spaces. Quaestiones Mathematicae. https://doi.org/10.2989/16073606.2019.1692092

24. Mengestie, T.: Carleson type measures for Fock-Sobolev spaces. Complex Anal. Oper. Theory 8(6), 1225-1256 (2014)

25. Mengestie, T.: Volterra type and weighted composition operators on weighted Fock spaces. Integr. Equ. Oper. Theory 76(1), 81-94 (2013)

26. Seyoum, W., Mengestie, T., Bonet, J.: Mean ergodic composition operators on generalized Fock spaces. RACSAM 114, 6 (2020). https://doi.org/10.1007/s13398-019-00738-w

27. Ueki, S.: Weighted composition operators on the Fock space. Proc. Am. Math. Soc. 135, 1405-1410 (2007)

28. Yosida, K.: Functional Analysis. Springer, Berlin (1978)

29. Yosida, K., Kakutani, S.: Operator-theoretical treatment of Markoff's Process and Mean Ergodic Theorem. Ann. Math. 42, 188-228 (1941)

Publisher's Note Springer Nature remains neutral with regard to jurisdictional claims in published maps and institutional affiliations. 